\title{
Particle-laden flow around an obstacle in a square pipe: experiments and modeling
}

\author{
Ouardia Ait Oucheggou ${ }^{1}$, Véronique Pointeau ${ }^{2}$, Guillaume Ricciardi ${ }^{2}$, Élisabeth Guazzelli ${ }^{3}$, \\ and Laurence Bergougnoux ${ }^{4, *}$ \\ ${ }^{1}$ Aix-Marseille Univ, CNRS, IUSTI, Marseille and CEA, DES, IRESNE, DTN, Cadarache, France \\ ${ }^{2}$ CEA, DES, IRESNE, DTN, Cadarache, France \\ 3 Aix-Marseille Univ, CNRS, IUSTI, Marseille and Université de Paris, CNRS, Matière et Systèmes Complexes (MSC) \\ UMR 7057, Paris, France \\ ${ }^{4}$ Aix-Marseille Univ, CNRS, IUSTI, Marseille, France
}

Received: 8 November 2019 / Accepted: 3 June 2020

\begin{abstract}
Particle trapping and deposition around an obstacle occur in many natural and industrial situations and in particular in the nuclear industry. In the steam generator of a nuclear power plant, the progressive obstruction of the flow due to particle deposition reduces the efficiency and can induce tube cracking leading to breaking and damage. The steam generator then loses its role as a safety barrier of the nuclear power plant. From a fundamental standpoint, dilute and concentrated particulate flows have received a growing attention in the last decade. In this study, we investigate the transport of solid particles around obstacles in a confined flow. Experiments were performed in a simplified configuration by considering a laminar flow in a vertical tube. An obstacle was inserted at the middle height of the tube and neutrally-buoyant particles were injected at different locations along the tube. We have investigated first the trajectories of individual particles using particle tracking (PT). Then, the particle trajectories were modeled by using the Boussinesq-Basset-Oseen equation with a flow velocity field either measured using particle image velocimetry (PIV) or calculated by the Code_Saturne software in order to account for the three-dimensional (3D) character of the obstacle wake. This paper presents a comparison between the experimental observations and the predictions of the modeling for an obstacle consisting of a rectangular step at a Reynolds number of $\approx 100$ and evidences the importance of accounting for the $3 \mathrm{D}$ complex nature of the flow.
\end{abstract}

Keywords: Particle-laden flow / obstacle / wakes

\section{Introduction}

About $11 \%$ of the world electricity is generated by 450 nuclear power reactors. Most of these nuclear power plants (65\% of them) are Pressurized Water Reactors (PWR). The Steam Generator (SG) is a crucial component of the PWR where the heat exchange between the primary and secondary circuits produces steam which is used to drive a turbine linked to an alternator to produce electricity. The tube bundle in the SG comprises 3300 to 6000 tubes supported at different heights by internal structures called Tube Support Plates (TPS). Clogging, i.e. the progressive obstruction mainly due by deposits of iron oxides, occurs in the quatrefoil holes between the tubes and the TPS designed for the circulation of the fluid. This phenomenon can have important consequences on the safety of the PWR. Therefore, the understanding of the origin of this mechanism $[1,2]$, especially that

\footnotetext{
* e-mail: laurence.bergougnoux@univ-amu.fr
}

related to thermal, chemical, hydrodynamic, and physical issues, requires particular attention. Investigations inside the PWRs are limited because of the difficulty to access to specific location of this phenomenon. The difficulty of undertaking experiments and instrumentation under thermohydraulic (pressure $\simeq 6 \mathrm{MPa}$, temperature $\simeq 280^{\circ} \mathrm{C}$ ) and chemical representative conditions in the SG justifies to simplify the problem and to perform model experiments. The objective of the present study is thus to examine the flow of particles around obstacles (which mimic the TPSs) and in particular to study their preferential locations of trapping. To simplify further the problem, we have chosen to focus on the hydrodynamic effects and to neglect other phenomena such as thermal and chemical interactions.

From a fundamental standpoint, the flow around a bluff body is controlled by several parameters: the shape of the obstacle, the environment in which the body is located (a confined pipe [3] or a free surface channel $[4])$, and the flow Reynolds number, $R e_{f}$. Pressure losses 
caused by a sudden change in the flow section give rise to recirculation zones. This is the case of a backwardfacing step flow which has been extensively studied and used as a test case in numerical simulations. Experimental investigations of laminar, transitional, and turbulent flows with regions of separation behind a two-dimensional backward-facing step were carried out by Armaly et al. [5] with an air flow in a pipe with a blockage ratio of 0.5. The length of the wake behind the step was measured, as well as the lengths and locations of the other recirculating-flow regions. One of the rare studies focusing on the upstream region of an obstacle has been conducted by Hattori et al. [6]. This numerical study shows that the size of the recirculation zone upstream of the forward-facing step becomes smaller with increasing $R e_{f}$. Another result is that the obstacle blockage in the pipe influences the sizes of the recirculation zones.

Dilute and concentrated particulate flows have received a growing attention in the last decade. For example, the study conducted by Matas et al. [7] on dilute suspensions of neutrally buoyant particles in Poiseuille flow extended that of Segrè and Silberberg [8] and showed that particles accumulate on a narrow annulus which moves toward the wall as $R e_{f}$ increases. The same authors [9] investigated the influence of suspended particles on the transition to turbulence in a pipe flow. Their results indicate that particle sizes and concentration influence this transition: small particles cause a delay in transition to larger critical Reynolds number while, for larger particles at low concentrations, the opposite effect is observed.

In the case of suspension flows in obstructed pipes, the studies are mostly focused on the downstream side of the obstacle, although the area affected by clogging is the upstream region of the obstacles in the practical case of steam generators. The behavior of monodisperse and neutrally buoyant particles in the wake of a cylinder has been examined by Haddadi et al. [10] at moderate $R e_{f}<30$. Their experimental observations in a microfluidic device indicated the formation of a particle-depleted region in the wake of the obstacle. Using simulations based on a lattice-Boltzmann method, they investigated the behavior of a single particle placed at different initial locations. They found that, when released inside the wake region, the particles move towards the boundary of the wake until reaching a limit-cycle trajectory. When many particles are introduced into the wake, because of particle hydrodynamic interactions, particles can move from one lobe of the wake to the other, or completely exit the wake by escaping into the free stream. Recently, the same authors [11] examined numerically the trapping of particles in the wake of obstacles having different geometries by varying the size of particles and the density ratio between the particles and the fluid. They showed that the vortex depopulation kinetics depends upon the shape of the obstacle as well as upon the density ratio of the particle and the fluid and the size ratio between the particles and the obstacle.

The particle trapping or deposition phenomenon in particle-laden flow inside confined geometries is still poorly understood. In this paper, an experimental investigation of the behavior of spherical particles around an obstacle in laminar flow is presented. Experimental particle trajectories are compared to predictions obtained by using the equation of motion for a small rigid sphere at low Reynolds numbers $[12,13]$.

\section{Materials and methods}

\subsection{Dimensional analysis}

Before presenting the experimental setup, the different physical and geometrical quantities of the study are discussed. The square pipe is characterized by its hydraulic diameter, $d_{h}$, defined as the ratio of four times the crosssectional area of the flow and the wetted perimeter of this cross-section. The fluid flowing through the tube is characterized by its viscosity, $\mu$, its density, $\rho_{f}$, and the inlet velocity, $u_{0}$. The particles are spherical with a radius, $a_{p}$, and a density, $\rho_{p}$. An additional quantity is the gravitational acceleration, $g$. We thus have seven independent physical quantities. Using the Buckingham $\pi$ theorem, dimensionless analysis indicates that the present problem is characterized by four dimensionless parameters: the velocity ratio, $W=v_{S t} / u_{0}$, where $v_{S t}=2 a_{p}^{2}\left(\rho_{p}-\rho_{f}\right) g / 9 \mu$ is the Stokes velocity of particles, the Stokes number, $S t=\frac{2}{9}\left(\rho_{p}+\rho_{f} / 2\right) a_{p}^{2} u_{0} / \mu d_{h}$, characterizing the behavior of particles suspended in a fluid flow (a particle with a low Stokes number follows fluid streamlines while a particle with a large Stokes number is dominated by its inertia), the density ratio, $R=\rho_{f} /\left(\rho_{p}+\rho_{f} / 2\right)$, and the size ratio, $P=a_{p} / d_{h}$, which is always rather small. By combining these four parameters, two Reynolds numbers can be obtained: the Reynolds number of the particles, $R e_{p}=v_{s t} \rho_{f} a_{p} / \mu=\frac{2}{9} S t W R / P$, and the Reynolds number of the fluid, flow, $R e_{f}=u_{0} \rho_{f} d_{h} / \mu=R e_{p} /(P W)$.

\subsection{Experimental setup}

Experiments are performed in a transparent Plexiglass ${ }^{\circledR}$ pipe having an inner square cross-section of $2.5 \times 2.5 \mathrm{~cm}^{2}$ with $d_{h}=2.5 \mathrm{~cm}$, and a filled height of $\approx 100 \mathrm{~cm}$ obstructed by a rectangular obstacle inserted at the middle height of the tube, as sketched in Figure 1. All the experiments are performed at the ambient room temperature of $23 \pm 1{ }^{\circ} \mathrm{C}$. The fluid is a mixture of water and saccharose $(13.5 \%)$ with a density $\rho_{f}=1049 \mathrm{~kg} \cdot \mathrm{m}^{-3}$ and a viscosity $\mu=1.5 \mathrm{mPa}$.s at $23 \pm 1{ }^{\circ} \mathrm{C}$. The upward flow in the pipe is provided by a pump (SIMATEC/MVPUMPSYSTEM) covering a range of flow up to $R e_{f}=$ 1000. The inlet fluid velocity is fixed at $u_{0}=0.54 \mathrm{~cm} . \mathrm{s}^{-1}$, i.e. $R e_{f}=94$. Spherical particles made of polystyrene having a radius $a_{p}=250 \pm 5 \mu \mathrm{m}$ and density $\rho_{p}=$ $1050 \mathrm{~kg} . \mathrm{m}^{-3}$ are used.

A digital camera (JAI SP-12000M-CXP4-F-XT, $4096 \times$ 700 pixels, $120 \mathrm{fps}$ ) placed in front of the tube is focused on the obstacle and the particles. The flow characterization is performed by a non-intrusive method, particle image velocimetry (PIV) using the Matlab ${ }^{\mathrm{TM}}$ PIV software DPIVsoft [14]. The fluid is seeded with hollow particles used as fluid tracers (Dantec Measurement Technology) having a diameter $\approx 15 \mu \mathrm{m}$ and a density 


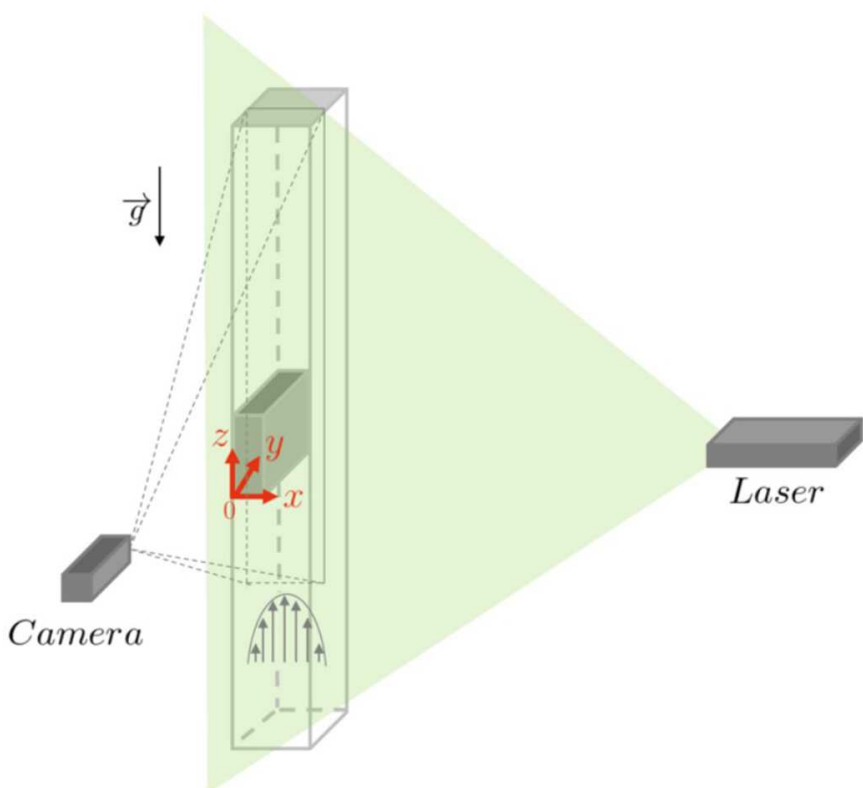

Fig. 1. Schematic view of the experimental setup: the pipe has an inner square cross-section of $2.5 \times 2.5 \mathrm{~cm}^{2}$ with $d_{h}=2.5 \mathrm{~cm}$, and a filled height of $\approx 100 \mathrm{~cm}$ obstructed by a rectangular obstacle inserted at the middle height of the tube.

$\approx 1400 \mathrm{~kg} \cdot \mathrm{m}^{-3}$. For all PIV measurements, the spatial resolution is $0.13 \times 0.13 \mathrm{~cm}^{2}$, corresponding to an interrogation window of $32 \times 32$ pixels with an overlap of $50 \%$. The error in the measurements of the time-averaged velocity fields was estimated to remain below $10 \%$.

Particle tracking (PT) is used to track the motion of particles and infer their velocities. A neon light illuminates the region around the obstacle and the digital camera records the particle motions at 15 frames/s. The particle tracking scheme uses the plugin MTrackJ of the ImageJ software [15]. The experimental error in the particle center measurement is $\approx 1 / 5$ of the particle diameter. Since the camera is at the same location in the PIV and PT measurements, the coordinates of the particle can be accurately positioned inside the velocity-vector map of the flow as a function of time.

\subsection{Simulation with Code_Saturne}

Code_Saturne is a free, open-source software developed and released by EDF to solve computational fluid dynamics (CFD) applications. It solves the Navier-Stokes equations for 2D, 2D-axisymmetric, and 3D flows, steady or unsteady, laminar or turbulent, incompressible or weakly dilatable, isothermal or not, with scalar transport if required. In this study, a $3 \mathrm{D}$ steady simulation of the flow is performed. A structured hexahedral mesh is used with cell sizes of $1 \times 1 \times 1 \mathrm{~mm}^{3}$ along the tube dimensions. The mean flow is in the $z$-direction (opposite to the gravity direction) with an inlet uniform velocity $u_{0}=0.54 \mathrm{~cm} \cdot \mathrm{s}^{-1}$. Computational convergence is verified by following the velocities of the flow $\left(u_{x}, u_{y}, u_{z}\right)$ throughout the iterations. Convergence is obtained after 200 iterations.

\subsection{Modeling}

We follow the same approach as that developed by Bergougnoux et al. [16] in their study of particle sedimentation in a cellular flow field at low Stokes numbers $(<0.1)$. Bergougnoux et al. performed experiments with Poly(methyl methacrylate) (PMMA) spherical particles sedimenting in a cellular flow. A model inspired by that of Maxey [17] was developed by considering the additional effect of the particle history force but also the modification of the drag force of the particle when the particle Reynolds $R e_{p}$ increases. This modeling is based on the resolution of the Boussinesq-Basset-Oseen equation (BBO) including Faxén corrections to consider the influence of the particle size $[12,13]$. The BBO equation predicts the acceleration $d \boldsymbol{v} / d t$ and the velocity $\boldsymbol{v}$ of the particle knowing its initial position and the velocity field $\boldsymbol{u}=\left(u_{x}, u_{y}, u_{z}\right)$ of the fluid:

$$
\begin{aligned}
m_{p} \frac{d \boldsymbol{v}}{d t} & =\left(m_{p}-m_{f}\right) \boldsymbol{g}-6 \pi \mu a_{p}\left(\boldsymbol{v}-\boldsymbol{u}-\frac{1}{6} a_{p}^{2} \nabla^{2} \boldsymbol{u}\right) \\
& +m_{f} \frac{D \boldsymbol{u}}{D t}-\frac{1}{2} m_{f} \frac{d \boldsymbol{v}}{d t}+\frac{1}{2} m_{f} \frac{D}{D t}\left(\boldsymbol{u}+\frac{1}{10} a_{p}^{2} \nabla^{2} \boldsymbol{u}\right)
\end{aligned}
$$

where $m_{p}=\frac{4}{3} \pi a_{p}^{3} \rho_{p}$ and $m_{f}=\frac{4}{3} \pi a_{p}^{3} \rho_{f}$, are respectively the particle mass and the mass of the displaced fluid. In this equation $(1), d / d t$ denotes the time derivative in a frame moving with the particle, while $D / D t$ the derivative following the fluid element. The forces included in the right-hand side of equation (1) are the buoyancy force, the Stokes drag, the fluid acceleration, and the added mass effect. The history Boussinesq-Basset force has been neglected in the present study. The terms in $a_{p}^{2} \nabla^{2} \boldsymbol{u}$ are the Faxén corrections.

We can consider that the flow field $\boldsymbol{u}$ is steady and write the dimensionless form $\left(^{*}\right)$ of equation (1), using $u_{0}$ as the velocity scale and $d_{h}$ as the length scale,

$$
\begin{aligned}
\frac{d \boldsymbol{v}^{*}}{d t^{*}}= & \frac{1}{S t}\left(W \frac{\boldsymbol{g}}{\|g\|}+\boldsymbol{u}^{*}-\boldsymbol{v}^{*}+\frac{P^{2}}{6} \nabla^{2} \boldsymbol{u}^{*}\right) \\
& +R\left(\frac{3}{2} \boldsymbol{u}^{*} \cdot \nabla \boldsymbol{u}^{*}+\frac{P^{2}}{20} \boldsymbol{u}^{*} \cdot \nabla\left(\nabla^{2} \boldsymbol{u}^{*}\right)\right) .
\end{aligned}
$$

For the selected combination of particles and fluid, we have $W=0.019, S t=0.003, R=0.666$, and $P=0.011$. The Faxén corrections, proportional to $P^{2}$, are negligible and the drag-buoyancy term, inversely proportional to $S t$, largely prevails over the other terms as also found by Bergougnoux et al. [16].

\section{Results and discussion}

\subsection{Flow characterization}

\subsubsection{Experimental characterization}

The flow characterization is obtained experimentally using PIV in a plane $\left(x^{*}, z^{*}\right)$ by considering 500 pairs of 


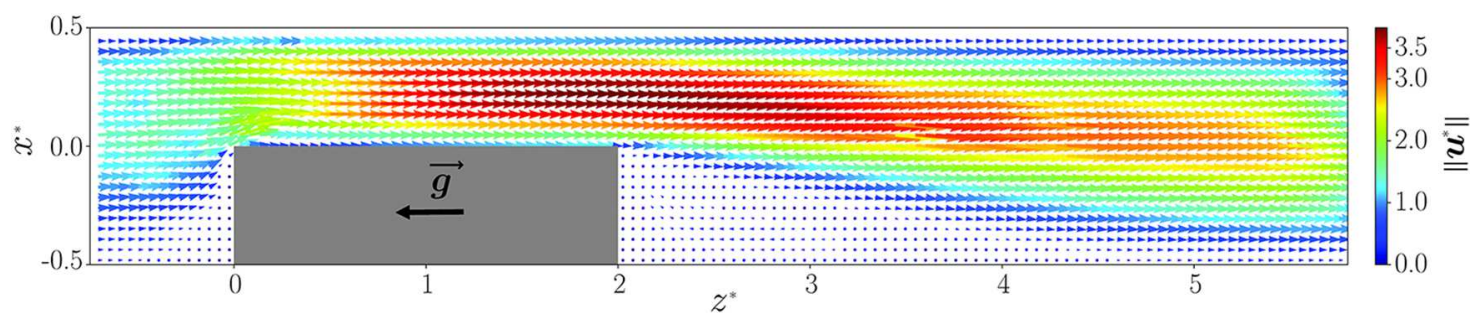

Fig. 2. Velocity field $\left(u_{x}^{*}, u_{z}^{*}\right)$ measured by PIV in the $\left(x^{*}, z^{*}\right)$ plane at $y^{*}=0.5$.

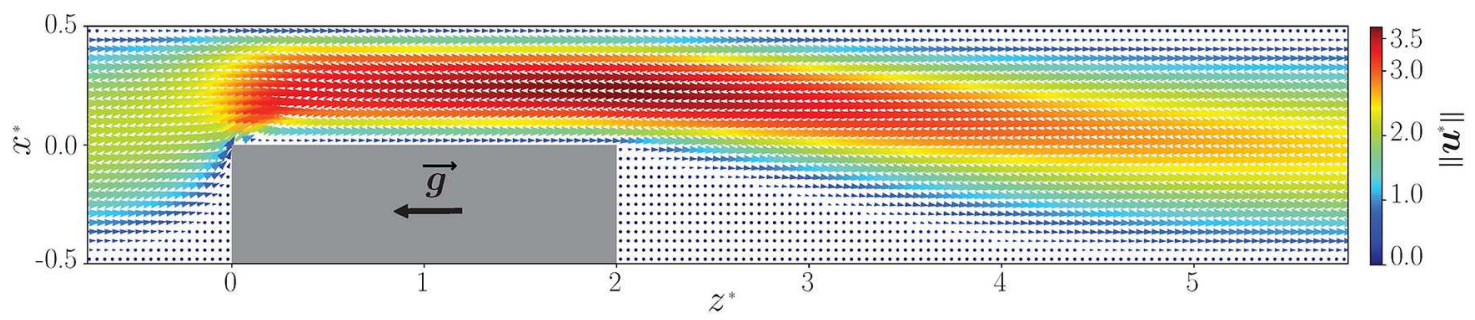

Fig. 3. Velocity field calculated by Code_Saturne in the $\left(x^{*}, z^{*}\right)$ plane, $y^{*}=0.5$.

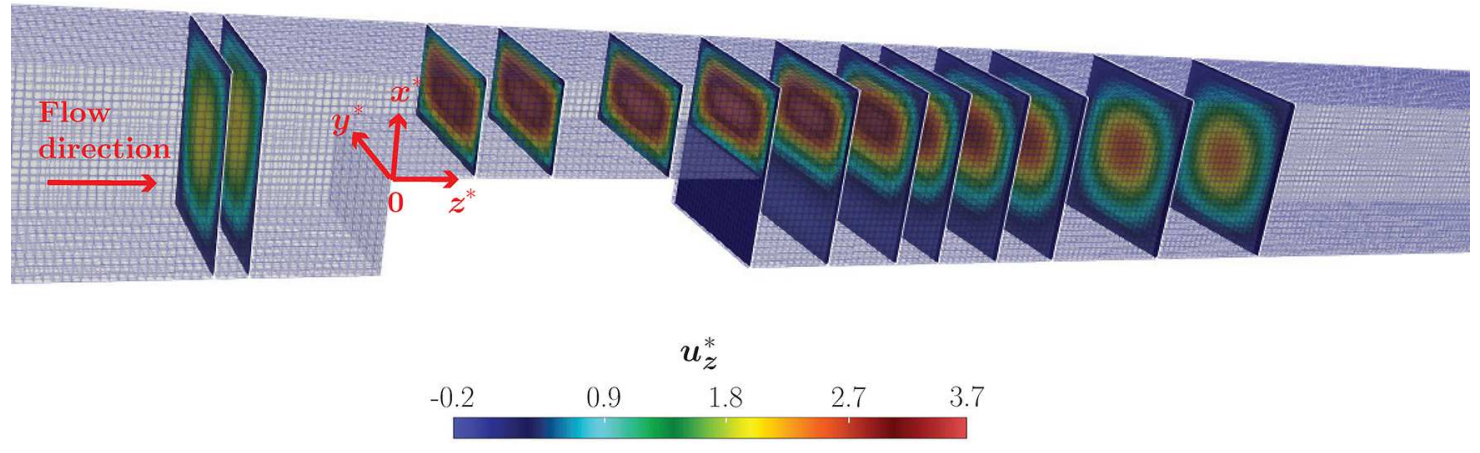

Fig. 4. Slices of $u_{z}^{*}$ velocity calculated by Code_Saturne in different $\left(x^{*}, y^{*}\right)$ planes.

snapshots. From each pair of snapshots, one instantaneous velocity field can be determined. In this steady-state regime, the time-averaged velocity field $\left(u_{x}^{*}, u_{z}^{*}\right)$, in the center section $\left(y^{*}=0.5\right)$ is presented in Figure 2 .

In this Figure 2, recirculation zones are formed upstream and downstream from the obstacle which is a classical result found in the literature. There are some discontinuity zones in the velocity field before reaching the obstacle, due to a lack of seeding in these regions. The fluid is accelerating when passing above the obstacle $\left(u^{*} \approx 4 u_{0}\right)$ due to flow rate conservation. In the recirculation zones, the fluid velocity is weak: particles can be trapped in these areas. In the wake of the obstacle, the main recirculation zone is clearly visible.

\subsubsection{Numerical characterization}

Because of the complexity of the flow around the obstacle, access to the third velocity component of the flow, $u_{y}$, is essential. The 3D flow characterization is obtained using Code_Saturne. The velocity field $\left(u_{x}^{*}, u_{z}^{*}\right)$ computed in the same plane $\left(y^{*}=0.5\right)$ as that of the experimental result is plotted in Figure 3 and shows a decent agreement with the experimental velocity field of Figure 2.
Figure 4 presents sliced views of the flow field. It exhibits the Poiseuille velocity profile in the first two planes before the obstacle and the two last planes towards the outlet. The flow complexity and the importance of the velocity field $\left(u_{x}^{*}, u_{y}^{*}\right)$ in different planes $\left(x^{*}, y^{*}\right)$ are reported in Figure 5. This last figure shows that the component $u_{y}^{*}$ is not negligible. Clearly, there is a three dimensional structure of the flow as evidenced for instance on the top of the obstacle for graph (d). The numerical 3D flow obtained by the Code_Saturne will then be used for solving equation (2) and comparing to the experiments.

\subsection{Particle trajectories}

\subsubsection{Experimental trajectories}

Figure 6 presents three experimental trajectories obtained by $\mathrm{PT}$ as well as the results of the modeling with equation (2) when using the velocity field $\left(u_{x}^{*}, u_{z}^{*}\right)$ given by PIV measurements. The particles are experimentally injected at different times. The first particle $(\square,-,-)$ released in the free stream passes over the obstacle and remains away from the recirculation regions. The comparison with the modeling shows a good agreement. 

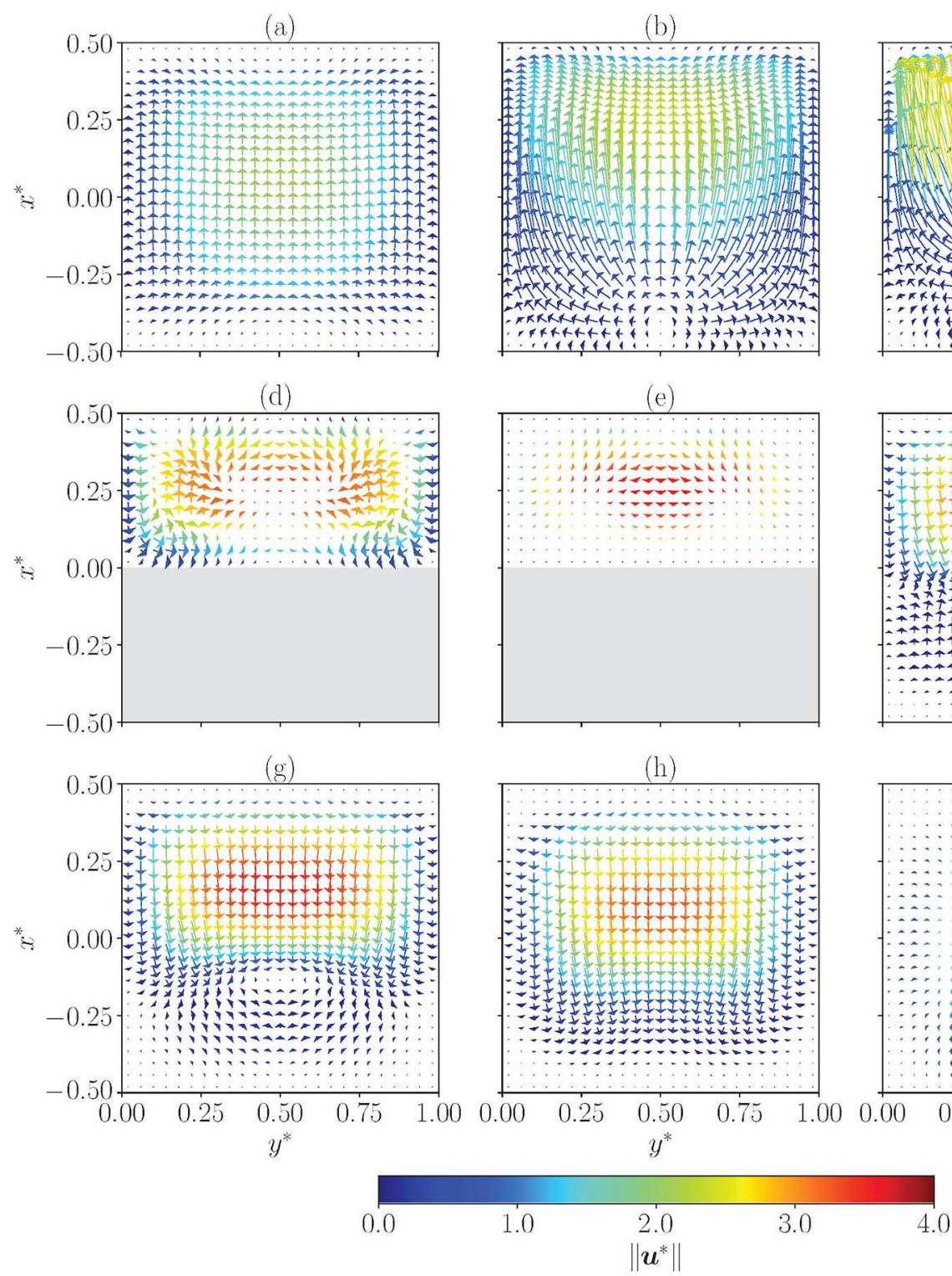

(b)

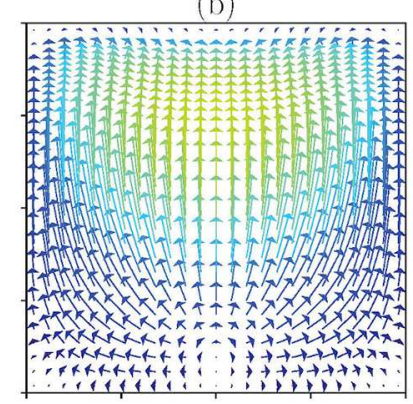

(e)

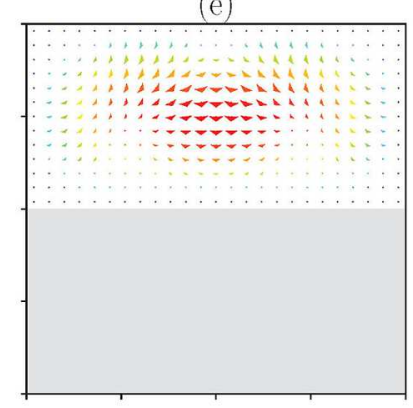

(h)

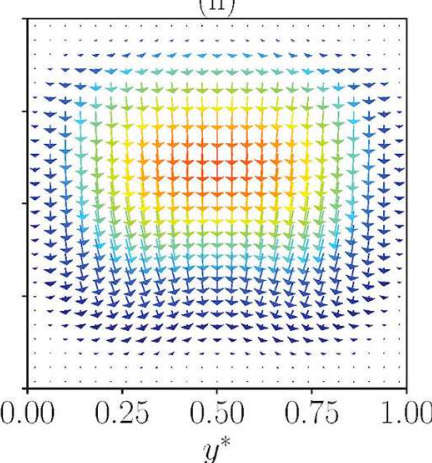
4.0 (c)

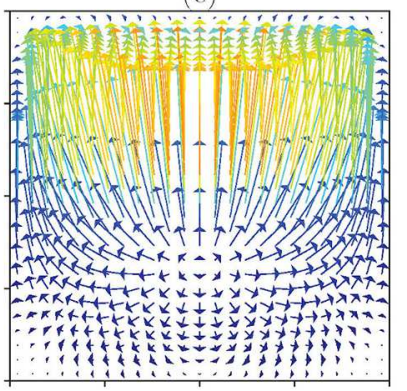

(f)

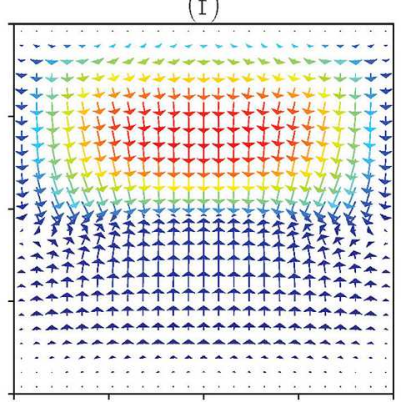

(i)

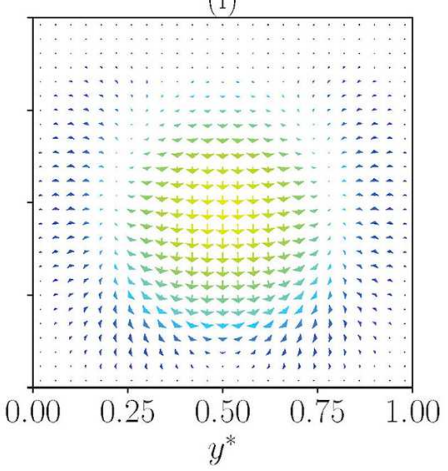

Fig. 5. Velocity field calculated by Code_Saturne in different $\left(x^{*}, y^{*}\right)$ planes at (a) $z^{*}=-0.78,(\mathrm{~b}) z^{*}=-0.22,(\mathrm{c}) z^{*}=-0.02$, (d) $z^{*}=0.42$, (e) $z^{*}=1.46$, (f) $z^{*}=2.10$, (g) $z^{*}=2.50$, (h) $z^{*}=3.22$ and (i) $z^{*}=5.62$.

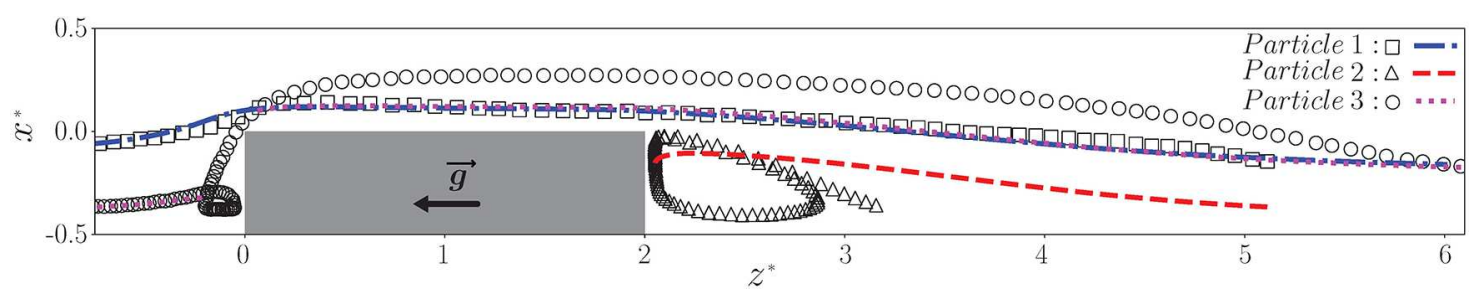

Fig. 6. Experimental trajectories of three particles $(\square, \triangle, \circ)$ and modeling results using the PIV flow field (-.-, ,--, ...).

Conversely, for particles injected in the downstream wake $(\triangle,--)$ or upstream of the obstacle $(\circ, \ldots)$, the model cannot capture properly the trajectories because of the $3 \mathrm{D}$ character of the flow in these recirculation zones which is not given by the 2D PIV flow field.

\subsubsection{Modeling using the numerical 3D flow field}

In this section, we now use the $3 \mathrm{D}$ flow calculated by Code_Saturne to solve equation (2). The new predictions are compared with the experimental trajectories presented in Figure 6. 

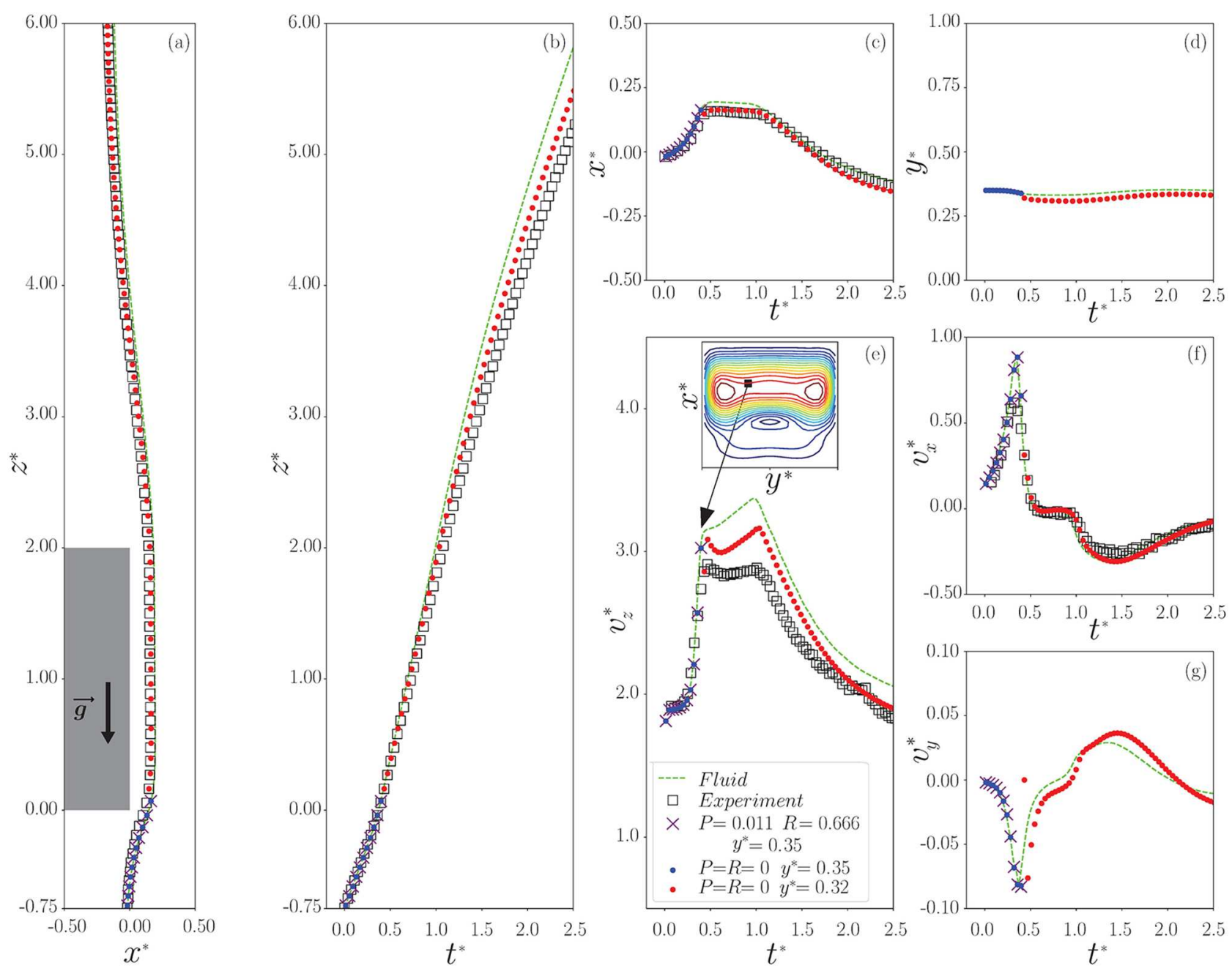

Fig. 7. (a) Experimental trajectory $(\square)$ and modeling prediction $(\bullet, \bullet)$ of particle 1; Temporal evolution of particle position (b) $z^{*}$, (c) $x^{*}$ and (d) $y^{*}$; Particle velocity (e) $v_{z}^{*}$, (f) $v_{x}^{*}$ and (g) $v_{y}^{*}$.

Figure 7 presents the results for the particle released in the free stream. It is mostly important for the numerical modeling to fix the coordinate $y^{*}$ at the initial time of the computation. We have taken $y^{*}=0.35(\bullet)$ from $t^{*}=0$ and $y^{*}=0.32(\bullet)$ from $t^{*} \approx 0.5$. The predictions with $P=0, R=0, W=0.019$, and $S t=0.003$ are in excellent agreement with the experimental data. We have also calculated the first part of the trajectory with $P=0.011$ and $R=0.666(\times)$. The comparison with the trajectory using $P=0$ and $R=0(\bullet)$ shows that these two parameters do not affect the particle trajectory. Therefore, the model is simplified by neglecting these terms in equation (2). We should also note that we computed the trajectory of a fluid particle having the same initial conditions and that the particle does not deviate significantly from being a fluid tracer.

We proceed similarly for the second particle injected in the wake shown in Figure 8. As soon as the model can no longer follow the experimental trajectory (due to the complexity of the fluid in the wake of the obstacle), we adjusted the value of the $y^{*}$ component. Good agreement between the predictions and the experimental data is found. The trajectory of a fluid particle has been also computed (--) with same initial positions. Clearly, the particle does not simply follow the flow lines and is not a simple fluid tracer.

The trajectory of the third particle injected upstream of the obstacle is shown in Figure 9. To be able to follow the particle, we had to change three times the plane $y^{*}(\bullet, \bullet$, •). Experimentally, the particle turns once when arriving in the recirculation zone upstream of the obstacle and then leaves by bypassing it. Figure 9d shows that the particle, in addition to a change of position in the observation plane $\left(x^{*}, z^{*}\right)$, also moves in the direction $y^{*}$. To follow the particle and to predict correctly its trajectory, it is essential to have a measure of its injection plane. Again this particle is not a simple fluid tracer, see the $(--)$ curve.

\section{Conclusion}

In this study, we have examined experimentally the dynamics of quasi-neutrally-buoyant particles around 

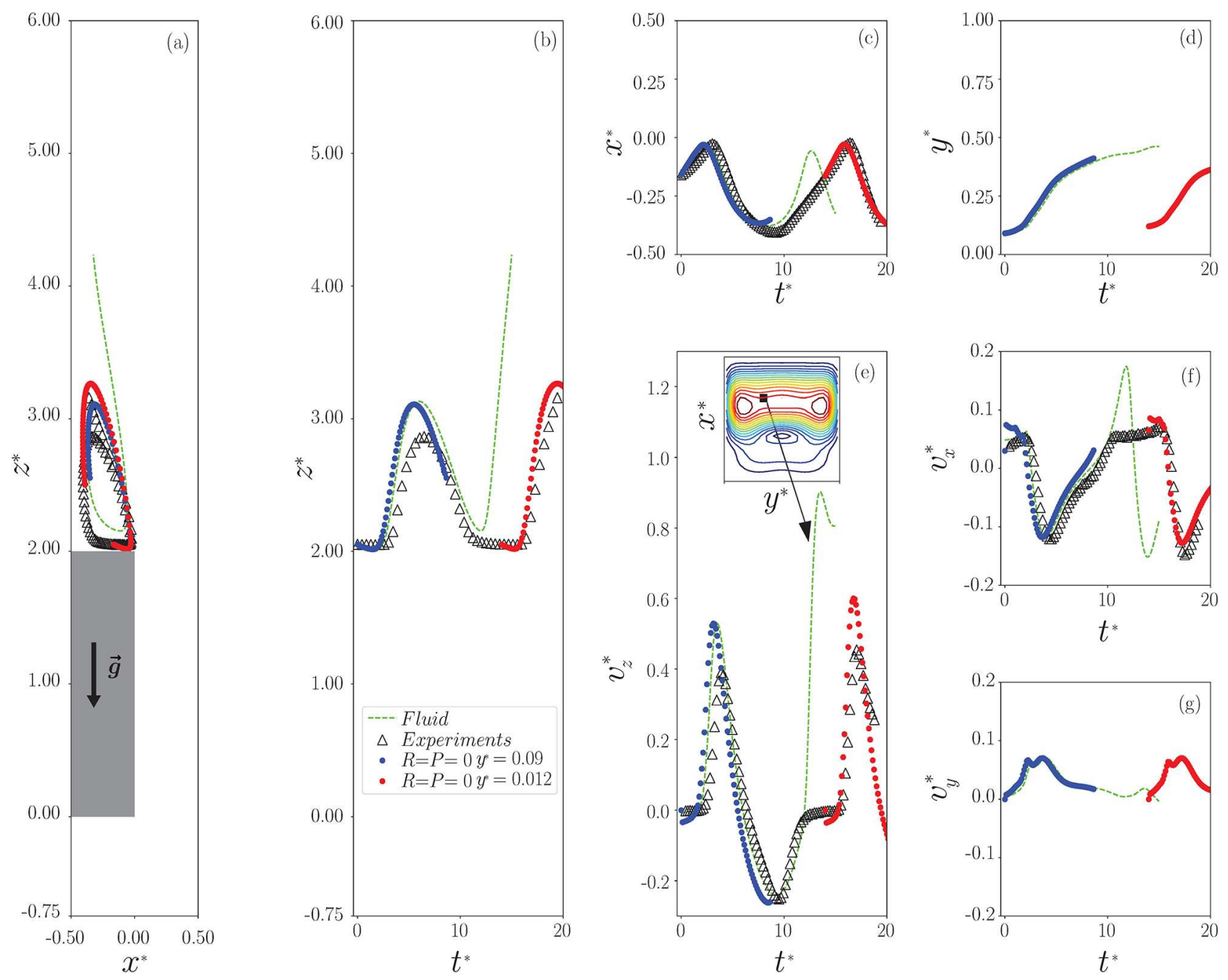

Fig. 8. (a) Experimental trajectory $(\triangle)$ and modeling prediction $(\bullet$, •) of particle 2; Temporal evolution of particle position (b) $z^{*}$, (c) $x^{*}$ and (d) $y^{*}$; Particle velocity (e) $v_{z}^{*}$, (f) $v_{x}^{*}$ and (g) $v_{y}^{*}$.

an obstacle. We have also used a model for the Lagrangian motion of particles at low Stokes number, $S t=310^{-3}$, and for $R e_{f} \approx 100$. The predicted trajectories have been compared to the experimental observations. The 3D character of the flow makes the problem complex, in particular in the recirculation zones upstream and downstream of the obstacle. Decent agreement between experiments and modeling can be achieved when the 3D nature of the flow is accounted for in a very fine manner.

\section{Nomenclature}

$\begin{array}{ll}a_{p} & \text { Particle radius } \\ d_{h} & \text { Hydraulic diameter of tube } \\ \rho_{f} & \text { Fluid density } \\ \rho_{p} & \text { Particle density } \\ \mu & \text { Dynamic viscosity }\end{array}$

$\begin{array}{ll}g & \text { Gravitational acceleration } \\ u, v & \text { Fluid and particle velocity } \\ u_{0} & \text { Inlet fluid velocity } \\ v_{S t} & \text { Particle Stokes velocity } \\ P & \text { Size ratio } \\ R & \text { Density ratio } \\ W & \text { Velocity ratio } \\ R e_{f} & \text { Reynolds number of the flow } \\ S t & \text { Stokes number } \\ (x, y, z) & \text { Coordinates } \\ * & \text { Dimensionless quantity }\end{array}$

The authors thank P. Cervetti, S. Noel, and F. Ratouchniak for technical assistance in the experimental setup realization. The work was supported in part by CEA. The thesis of O. Ait Oucheggou benefits from a joint funding by CEA and AixMarseille Université (contract AMU-CNRS-CEA V4322.001). 

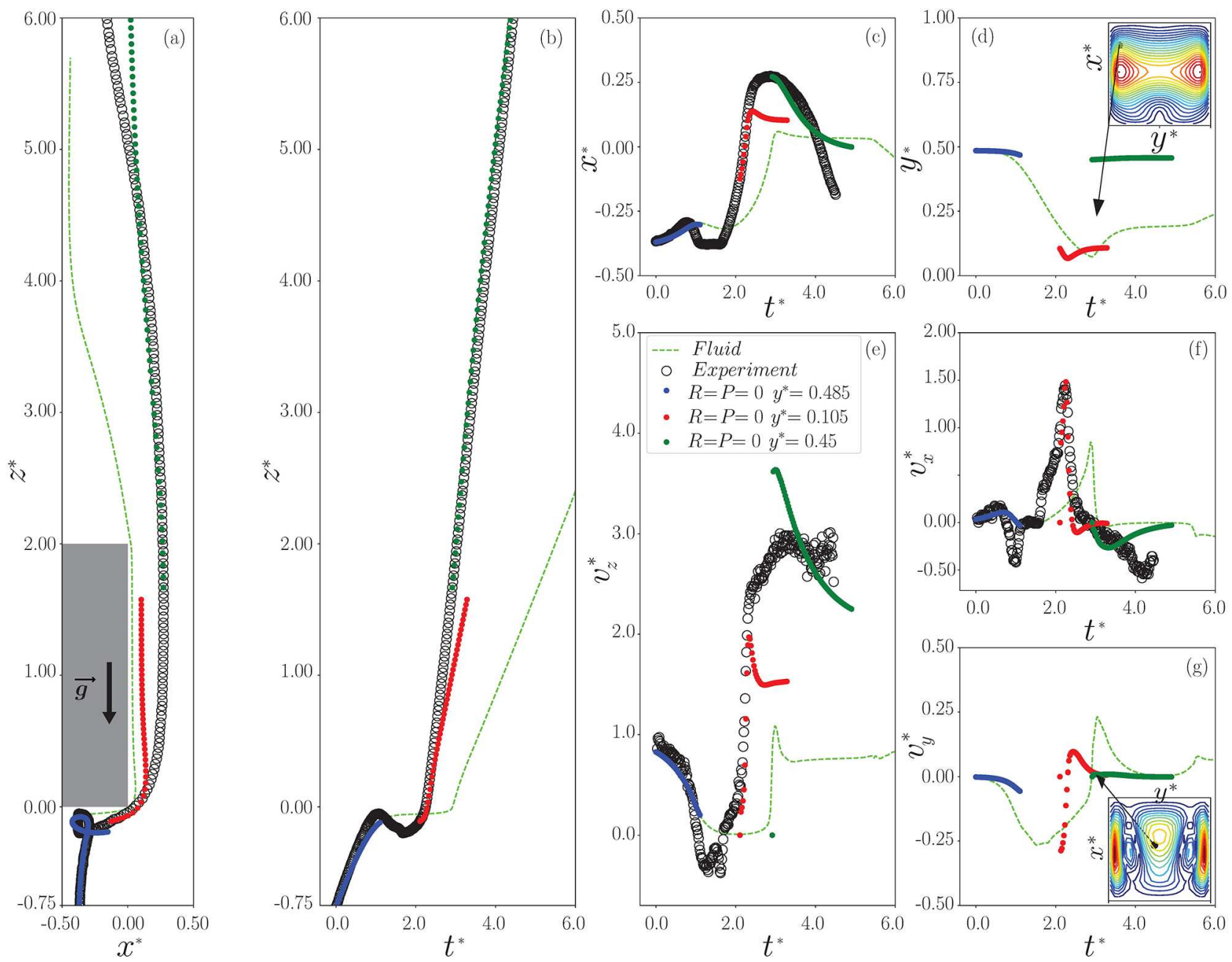

Fig. 9. (a) Experimental trajectory (o) and modeling prediction $(\bullet, \bullet)$ of particle 3 ; Temporal evolution of particle position $\left(\right.$ b) $z^{*}$, (c) $x^{*}$ and $(\mathrm{d}) y^{*}$; Particle velocity (e) $v_{z}^{*}$, (f) $v_{x}^{*}$ and $(\mathrm{g}) v_{y}^{*}$.

\section{References}

[1] G. Yang, V. Pointeau, E. Tevissen, A. Chagnes, A review on clogging of recirculating steam generators in pressurized water reactors, Prog. Nucl. Energy 97, 182-196 (2017)

[2] H. Rummens, J.T. Rogers, C.W. Turner, The thermal hydraulics of tube support fouling in nuclear steam generators, Nucl. Technol. 148, 268-286 (2004)

[3] L. Klotz, S. Goujon-Durand, J. Rokicki, J.E. Wesfreid, Experimental investigation of flow behind a cube for moderate Reynolds numbers, J. Fluid Mech. 750, 73-98 (2014)

[4] J.C.R. Hunt, C.J. Abell, J.A. Peterka, H. Woo, Kinematical studies of the flows around free or surface-mounted obstacles; applying topology to flow visualization, J. Fluid Mech. 86, 179-200 (1978)

[5] B.F. Armaly, F. Durst, J.C.F. Pereira, B. Schönung, Experimental and theoretical investigation of backward-facing step flow, J. Fluid Mech. 127, 473-496 (1983)

[6] H. Hattori, Y. Nagano, Investigation of turbulent boundary layer over forward-facing step via direct numerical simulation, Int. J. Heat Fluid Flow 31, 284-294 (2010)
[7] J.-P. Matas, J. F. Morris, É. Guazzelli, Inertial migration of rigid spherical particles in Poiseuille flow, J. Fluid Mech. 515, 171-195 (2004)

[8] G. Segré, A. Silberberg, Behaviour of macroscopic rigid spheres in Poiseuille flow Part 2. Experimental results and interpretation, J. Fluid Mech. 14, 136-157 (1962)

[9] J.-P. Matas, J. F. Morris, É. Guazzelli, Influence of particles on the transition to turbulence in pipe flow, Philos. Trans. Roy. Soc. A 361, 911-919 (2003)

[10] H. Haddadi, S. Shojaei-Zadeh, K. Connington, J.F. Morris, Suspension flow past a cylinder: particle interactions with recirculating wakes, J. Fluid Mech. 760, R2 (2014)

[11] H. Haddadi, S. Shojaei-Zadeh, J.F. Morris, LatticeBoltzmann simulation of inertial particle-laden flow around an obstacle, Phys. Rev. Fluids 1, 024201 (2016)

[12] R. Gatignol, The Faxén formulae for a rigid particle in an unsteady non-uniform Stokes flow, J. Mécaniq. Théor. Appl. 2, 143-160 (1983)

[13] M.R. Maxey, J.J. Riley, Equation of motion for a small rigid sphere in a non-uniform flow, Phys. Fluids 26, 883-889 (1983) 
[14] P. Meunier, T. Leweke, Analysis and treatment of errors due to high velocity gradients in particle image velocimetry, Exp. Fluids 35, 408-421 (2003)

[15] E. Meijering, O. Dzyubachyk, I. Smal, Methods for cell and particle tracking, Methods Enzymol. 504, 183-200 (2012)
[16] L. Bergougnoux, G. Bouchet, D. Lopez, É. Guazzelli, The motion of solid spherical particles falling in a cellular flow field at low Stokes number, Phys. Fluids 26, 093302 (2014)

[17] M.R. Maxey, The motion of a small spherical particles in a cellular flow field, Phys. Fluids 30, 1915-1928 (1987)

Cite this article as: O. Ait Oucheggou, V. Pointeau, G. Ricciardi, É. Guazzelli, L. Bergougnoux, Particle-laden flow around an obstacle in a square pipe: Experiments and modeling, Mechanics \& Industry 21, 517 (2020) 